\title{
Mobile CRISPR-Cas9 based anti-phage system in E. coli
}

\author{
Zhou Cao ${ }^{1,2 *}$, Yuxin Ma ${ }^{1,2 *}$, Bin Jia $(\bowtie)^{1,2}$, Ying-Jin Yuan ${ }^{1,2}$ \\ 1 Frontier Science Center for Synthetic Biology and Key Laboratory of Systems Bioengineering (Ministry of Education), Tianjin University, \\ Tianjin 300072, China \\ 2 Collaborative Innovation Center of Chemical Science and Engineering (Tianjin), School of Chemical Engineering and Technology, \\ Tianjin University, Tianjin 300072, China
}

(C) Higher Education Press 2022

\begin{abstract}
Escherichia coli is one of the most important microbial cell factories, but infection by bacteriophages in the environment may have a huge impact on its application in industrial production. Here, we developed a mobile CRISPR-Cas9 based anti-phage system for bacteriophages defense in E. coli. Two conjugative plasmids pGM1 (phosphoglucomutase 1) and pGM2 carrying one and two guide RNAs, respectively, were designed to defend against a filamentous phage. The results showed that the pGM1 and pGM2 could decrease the phage infection rate to $1.6 \%$ and $0.2 \%$ respectively in infected cells. For preventing phage infection in E. coli, the pGM2 decreased the phage infection rate to $0.1 \%$, while pGM1 failed to block phage infection. Sequence verification revealed that point mutations in protospacer or protospacer adjacent motif sequences of the phage genome caused loss of the defense function. These results support the potential application of MCBAS in E. coli cell factories to defend against phage infections.
\end{abstract}

Keywords phage infections, anti-phage, CRISPR-Cas9, conjugative transfer, synthetic biology

\section{Introduction}

With the development of synthetic biology, Escherichia coli has been cultivated for the bioproduction of a range of heterologous proteins [1,2], natural metabolites [3-5] and high-value chemicals. For example, it can be used to produce isobutanol [6], polyhydroxyalkanoates $[7,8]$, type II polyketide products [9] and avermectin [10]. However, some phages existing in nature can infect $E$. coli, resulting in decreases in cell density and the yield of target products

Received September 9, 2021; accepted November 3, 2021

E-mail: bin.jia@tju.edu.cn

${ }^{*}$ These authors contributed equally to this work. during fermentation, which may lead to enormous economic losses in industrial production [11].

Natural phages and bacteria are in a constant arms race that proceeds in continuous cycles of coevolution [12], which drives the development of diverse anti-phage strategies in bacteria and corresponding strategies to evade these anti-phage strategies in phages [13]. Phages interact with bacteria in the course of adsorption, infection and release [14]. In the adsorption process, bacteria can alter or disguise receptors through surface modification to elude phages [15]. For example, E. coli strains producing the K1 polysaccharide capsule can block phage T7 entry. However, K1-specific phages have evolved to counter this physical barrier [16]. In the infection process, bacteria can use a restriction-modification system to distinguish nonself-DNA from self-DNA and digest the former after phage injection [17]. Correspondingly, phages can protect their own DNA by accumulating point mutations at specific sites [18]. In a recent study, Zhou et al. reported that the diaminopurine $(Z)$ genome endows phages with evolutionary advantages to evade attacks by host restriction enzymes [19].

The clustered regularly interspaced short palindromic repeats associated protein (CRISPR-Cas) system provides 'adaptive' immunity through the generation of memories of past phage encounters that guide sequence-specific immunity [20]. The system uses guide RNAs (gRNAs) to specifically recognize phage DNA and cause cleavage to stop phage proliferation. However, some phages can escape the recognition of gRNAs by accumulating beneficial mutations, which cause loss of the immune function of the CRISPR-Cas system. Based on the high efficiency of sequencing technology and DNA synthesis technology, diverse gRNAs targeting the phage genome can reasonably be designed, which may enable bacteria to quickly acquire immunity against mutant phages or unreported phages.

In this work, we developed a mobile CRISPR-Cas9 based anti-phage system (MCBAS) for bacteriophages 
defense in E. coli. The MCBAS described here consists of a CRISPR-Cas9 system and the conjugation origin, which facilitates recognition of phage sequences and their delivery into $E$. coli before or after infection. As proof of concept (Fig. 1), MCBAS was used to defend against a filamentous MKR phage via both phage DNA clearance test and phage infection prevention test. Our results indicate that MCBAS carrying two gRNAs has better performance than that carrying a single gRNA. Sequence verification revealed that DNA mutations in the protospacer adjacent motif (PAM) or protospacer region of the phage genome caused loss of the defense function. Combining the highly programmable feature of gRNA targets and a broad host range by conjugation transfer, our method may provide a phage defense platform that can be extended to many other microbial cell factories.

\section{Experimental}

\subsection{Microbial strains and culture medium}

Plasmid cloning work was performed in the E. coli JM109 strain. All recipient strains used in this work were derived from the E. coli XL1-Blue strain. E. coli cells were cultured in Luria-Bertani (LB) broth media $\left(10 \mathrm{~g} \cdot \mathrm{L}^{-1}\right.$ peptone, $10 \mathrm{~g} \cdot \mathrm{L}^{-1} \mathrm{NaCl}, 5 \mathrm{~g} \cdot \mathrm{L}^{-1}$ yeast extract). $\mathrm{LB}$ solid medium was added with $15 \mathrm{~g} \cdot \mathrm{L}^{-1}$ agar. Kanamycin (Kan, $50 \mathrm{mg} \cdot \mathrm{mL}^{-1}$ ), tetracycline (Tet, $10 \mathrm{mg} \cdot \mathrm{mL}^{-1}$ ) and chloramphenicol $\left(\mathrm{Cm}, 10 \mathrm{mg} \cdot \mathrm{mL}^{-1}\right)$ were added when necessary.

\subsection{Plasmid construction}

All plasmids were constructed using standard molecular cloning techniques and Gibson assembly. Restriction endonucleases, T4 DNA ligase and Phusion polymerase chain reaction (PCR) kits from New England BioLabs (NEB) were used. PCR was performed with an ABI Thermal Cycler. Primers were synthesized by Tsingke. All plasmids were isolated with TIANprep Mini Plasmid Kits. The pGM0 was generated by inserting a green fluorecent protein (GFP) and oriT into the BBa K1218011BB from the Registry of Standard Biological Parts. The MKR phage was generated by inserting red fluorecent protein (RFP) into the $\mathrm{M} 13 \mathrm{KO} 7$ from NEB. Plasmid constructs were verified by restriction digests and sequencing by Tsingke Biotechnology Company. Plasmid map of MKR and pGM0 (phosphoglucomutase 0), pGM1 and pGM2 were shown in Figs. S1-S4 (cf. Electronic Supplementary Material, ESM).

\subsection{Conjugation}

Overnight cultures of $E$. coli JM109 donor cells containing MCBAS plasmids and recipient strains of XL1-Blue grown separately in LB medium with appropriate antibiotics were subcultured 1:100 and grown to an optical density 600 (OD600) of 0.5 at $37^{\circ} \mathrm{C}$. Cells were harvested by centrifugation at $5000 \times g$ for $5 \mathrm{~min}$, washed twice and then resuspended in LB medium without antibiotics. Equal volumes of the donor culture and the recipient culture were mixed and incubated for $1 \mathrm{~h}$ at $37^{\circ} \mathrm{C}$ for conjugation.

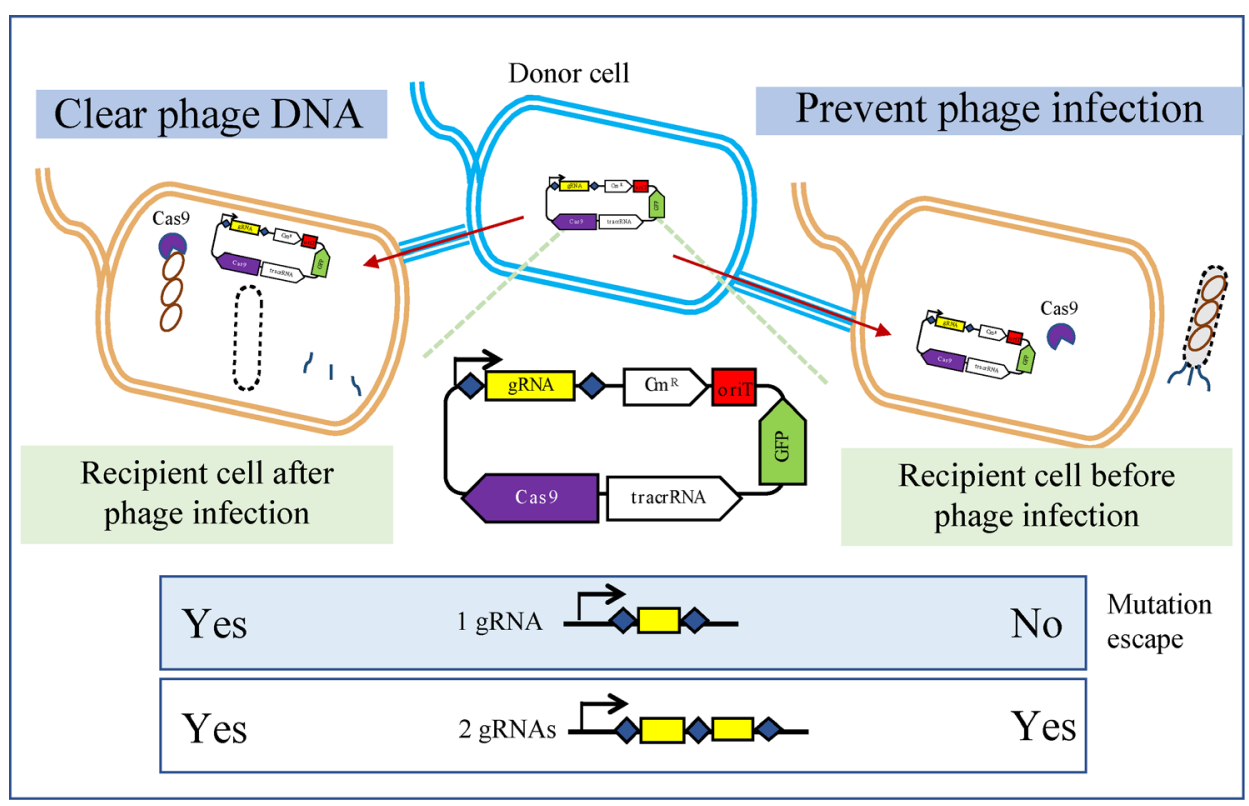

Fig. 1 A schematic representation of MCBAS for blocking phage infection. The MCBAS consist of CRISPR-Cas9 for cleavage of phage DNA and origin of transfer (oriT) for conjugation. To clear phage DNA in cells, the plasmids were delivered to recipient cells infected by phage via conjugation to remove phage DNA. To prevent phage infection, the CRISPR-Cas9 plasmids were delivered to recipient cells before phage infection via conjugation to prevent phage DNA replication. 
Samples were collected and vortexed for $10 \mathrm{~s}$ to stop conjugation, and then dilutions of the mixtures were spread on LB agar plates containing Tet and $\mathrm{Cm}$ to select transconjugant clones. The plates were incubated overnight at $37^{\circ} \mathrm{C}$.

\subsection{MKR phage preparation}

Colonies of the E. coli XL1-Blue strain containing MKR phages were inoculated into LB (Kan) medium and grown overnight at $37{ }^{\circ} \mathrm{C}$ and $220 \mathrm{r} \cdot \mathrm{min}^{-1}$. The culture was centrifuged at $12000 \mathrm{r} \cdot \mathrm{min}^{-1}$ for $5 \mathrm{~min}$, and the supernatant was filtered through a $0.2 \mu \mathrm{m}$ microfiltration membrane for sterilization. The filtered liquid was the prepared MKR phage solution, and its titer was approximately $10^{4}$ $\mathrm{PFU} \cdot \mu \mathrm{L}^{-1}$ (PFU is phages forming unit).

\subsection{Assay for clearing phage DNA in infected E. coli}

Equal volumes of the donor cell (E. coli JM109) culture and the recipient cell (E. coli XL1-Blue) culture were mixed and incubated in $1 \mathrm{~mL}$ of LB (Cm_Tet) medium for conjugate selection. Cell cultures were inoculated for $12 \mathrm{~h}$ at $37{ }^{\circ} \mathrm{C}$ and $900 \mathrm{r} \cdot \mathrm{min}^{-1}$. Ten microliters of bacterial cultures were removed every $2 \mathrm{~h}$ for 10 -fold dilution assays. The diluted bacterial solution was plated on LB $(\mathrm{Cm})$ and $\mathrm{LB}\left(\mathrm{Cm} \_\mathrm{Kan}\right)$ solid media in equal volumes and cultured at $37^{\circ} \mathrm{C}$. Each experiment was repeated three times.

\subsection{Assay for phage infection prevention}

The recipient cells containing MCBAS were generated by conjugation. Colonies were selected and inoculated into LB $\left(\mathrm{Cm} \_\right.$Tet $)$medium overnight at $37{ }^{\circ} \mathrm{C}$ and $220 \mathrm{r} \cdot \mathrm{min}^{-1}$, followe by subculturing at 1:100 and growth to an OD600 of 0.5 at $37^{\circ} \mathrm{C}$. Cultures of the recipient cells containing MCBAS were mixed with 2, 10, 50 and $100 \mu \mathrm{L}$ of MKR phage solution for infection. Cell cultures were inoculated for $12 \mathrm{~h}$ at $37{ }^{\circ} \mathrm{C}$ and $900 \mathrm{r} \cdot \mathrm{min}^{-1}$. Ten microliters of bacterial cultures were removed every $2 \mathrm{~h}$ for 10 -fold dilution assays. The diluted bacterial solution was plated on LB $(\mathrm{Cm})$ and LB $(\mathrm{Cm}$ Kan) solid media in equal volumes and cultured at $3 \overline{7}{ }^{\circ} \mathrm{C}$. Each experiment was repeated three times.

\subsection{Calculation of the MKR phage infection rate}

The diluted bacterial solution was plated on LB $(\mathrm{Cm})$ and LB $(\mathrm{Cm}$ Kan $)$ solid media in equal volumes and cultured at $37^{\circ} \mathrm{C}$. The number of single colonies on the two kinds of solid medium was counted after single colonies appeared. The number on LB $(\mathrm{Cm})$ solid medium represents the number of all $E$. coli cells in the corresponding diluted bacterial solution, while the number on LB (Cm_Kan) solid medium represents the number of E. coli cells infected by MKR phages. Therefore, the MKR phage infection rate is:

The colony number on LB (Cm_Kan) solid medium

The colony number on LB $(\mathrm{Cm})$ solid medium

$\times 100 \%$.

\section{Results and discussion}

\subsection{Design of MCBAS and MKR phage}

To increase the anti-phage ability of E. coli, we first designed conjugative CRISPR-Cas9 plasmids containing Cas9, trans-activating crRNA (tracrRNA), oriT, a GFP reporter gene and a $\mathrm{Cm}$ antibiotic resistance marker (Fig. 1). We then used the MCBAS to defend against a filamentous phage via both phage DNA clearance test and phage infection prevention test. To clear phage DNA in infected E. coli, MCBAS was delivered to infected recipient cells via conjugation to remove the phage DNA. To prevent phage infection in E. coli, the phage was added for infection after delivering MCBAS to uninfected recipient cells via conjugation. It is known that the M13 filamentous phages can only infect $E$. coli with the $\mathrm{F}$ factor in a nonlytic manner, which has contributed to their widespread use in phage display technology [21] and phage-assisted continuous evolution [22-24]. To simplify the calculation of phage infection rates, we engineered a new phage called MKR based on M13 filamentous phages (Fig. 2). The MKR has a Kan resistance gene and RFP gene for selection (Figs. 2(a) and $\mathrm{S} 1$ ). To assay the activity of the MKR phage, E. coli XL1Blue cells were mixed with the MKR phage and incubated in LB (Kan) medium. As shown in Fig. 2(b), red fluorescence was observed in E. coli after culturing overnight, demonstrating that the MKR phage had the ability to infect $E$. coli XL1-Blue cells. To calculate the MKR phage infection rate, appropriate MKR phage was used to infect $E$. coli XL1-Blue cells containing control plasmids without gRNA. As shown in Fig. 2(c), the infection rate reached approximately $76.6 \%$ within $2 \mathrm{~h}$ and reached $100 \%$ within $6 \mathrm{~h}$. These results demonstrate that the DNA of MKR can replicate and maintain a high level in the hosts. Additionally, MKR phages can reproduce in E. coli in a nonlytic manner.

\subsection{MCBAS clears phage DNA in E. coli}

To assay the anti-phage ability of the MCBAS, we first used them to clear the DNA of the MKR phage in infected E. coli (Fig. 3). As shown in Fig. 3(a), the CRISPR-Cas9 system should be transferred into from $E$. coli donor cell to recipient cell and gradually degrade the DNA of the MKR 
(a)

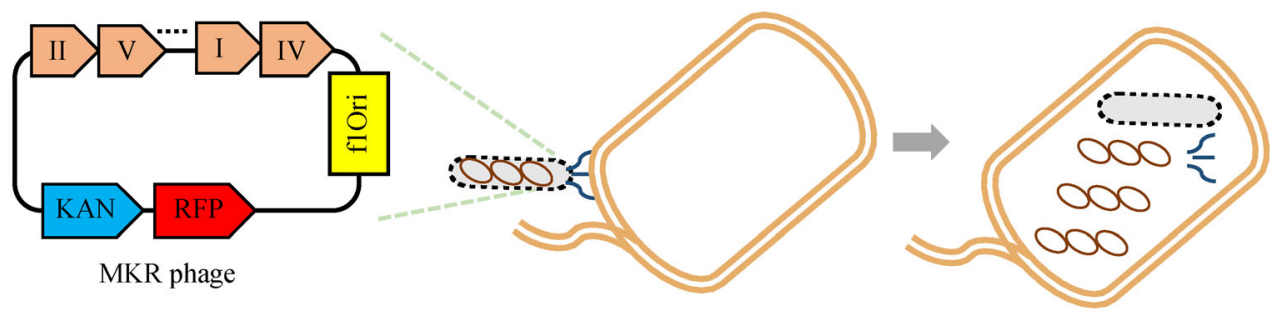

(b)

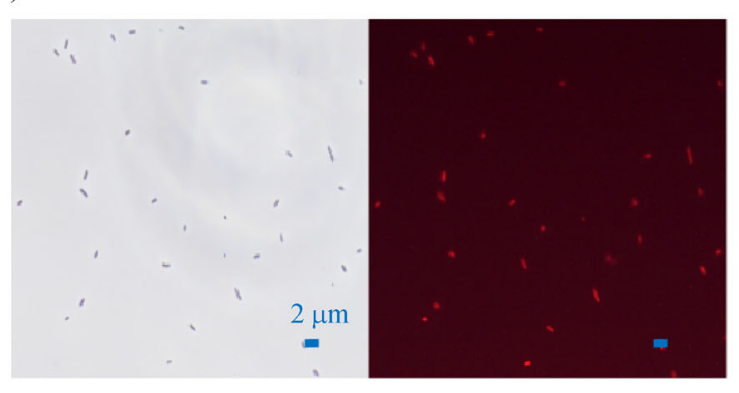

(c)

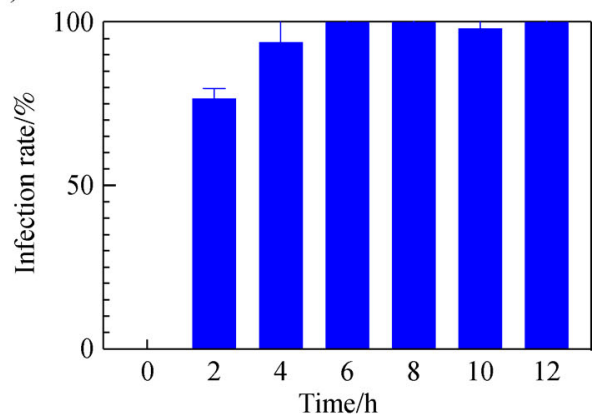

Fig. 2 Design of MKR phage. (a) Design of the MKR phage used to infect the E. coli XL1-Blue strain; (b) fluorescence images of the E. coli XL1-Blue strain infected by the MKR phage in the bright field (left) and red channel (right); (c) the MKR phage infection rate of the E. coli XL1-Blue strain after adding $2 \mu \mathrm{L}$ MKR phage (MOI (multiplicity of infection) $=1: 500$, error bars represent standard deviations (SDs) from three independent experiments).

phage. As shown in Fig. 3(b), MCBAS contained the transfer oriT, which should enable conjugative transfer of MCBAS plasmids from $E$. coli donor cell to recipient cell. To test the mobile ability of the MCBAS, the donor cell carrying pGM0 ( $\mathrm{Cm}$ resistance), the recipient cell carrying pUC19 (ampicillin (Amp) resistance) and mixture of the both strains were dropped on LB agars (Amp_Cm), respectively. As shown in Fig. 3(c), colonies were only observed in the mixture group, indicating the MCBAS could be conjugative transferred from $E$. coli donor cell to recipient cell. To test the degradation ability of MCBAS, we selected three targets on the DNA of the MKR phage: gRNA1 (tttgacgttggagtccacgttcttt), gRNA2 (tgtagcggegcattaagcgcggcgg) and gRNA3 (tttgacgttggagtccacgttcttt). We constructed the plasmid pGM1 containing the gRNA1 of tracrRNA and the plasmid pGM2 containing both gRNA2 and gRNA3 of tracrRNAs (Figs. S2-S4, cf. ESM). At first, the E. coli recipient cells were infected by the MKR phage, and then E. coli JM109 cells containing pGM0 (control), pGM1 and pGM2 were mixed with the recipient cells, respectively. The bacterial cultures were incubated for $12 \mathrm{~h}$, and phage yield assay was performed every $2 \mathrm{~h}$. As shown in Fig. 3(d), the infection rates of $E$. coli carrying pGM1 were decreased to $47.4 \%, 8.8 \%$ and $1.6 \%$ at 2,6 and $10 \mathrm{~h}$, respectively. The results showed that 98.4\% of the E. coli population had achieved MKR elimination after $12 \mathrm{~h}$ of culture. For E. coli carrying pGM2, the infection rate was significantly decreased to $2.3 \%$ at $2 \mathrm{~h}$ and below 3\% thereafter. The final infection rate of $E$. coli carrying pGM2 reached approximately
$0.2 \%$, indicating that both pGM1 and pGM2 were effective in blocking MKR phage replication in vivo. We also performed fluorescence imaging to assess the states of MKR phage DNA replication and the MCBAS distribution at $12 \mathrm{~h}$, which were indicated by RFP and GFP, respectively. As shown in Fig. 3(e), few E. coli cells with pGM1 or pGM2 expressed RFP, while all E. coli cells expressed GFP at $12 \mathrm{~h}$. The DNA of MKR phages was degraded by pGM1 and pGM2, which is consistent with the above phage yield assay. Therefore, these results demonstrated that pGM1 and pGM2 can clear the DNA of MKR phages in infected E. coli, and pGM2 was more effective in this study.

\subsection{MCBAS prevents phage infection of E. coli}

In addition, we also studied whether MCBAS can prevent MKR phages from infecting E. coli (Fig. 4). First, E. coli recipient cells were transferred with pGM0, pGM1 and pGM2 through conjugation, followed by infection with MKR phages (Fig. 4(a)). To test the preventive effect of the MCBAS, E. coli recipient cells carrying pGM0, pGM1 and pGM2 were mixed with MKR phages $(\mathrm{MOI}=1: 500)$. The bacterial cultures were incubated for $12 \mathrm{~h}$, and phage yield assay was performed every $2 \mathrm{~h}$. As shown in Fig. 4(b), the overall infection rates of $E$. coli carrying pGM1 were below $2 \%$ before $8 \mathrm{~h}$, increased to $15.2 \%$ at $10 \mathrm{~h}$, and reached $92.0 \%$ at 12 h. For E. coli carrying pGM2, all infection rates at each time point were below $1 \%$. These results indicate that pGM2 had a better prevention effect 
(a)

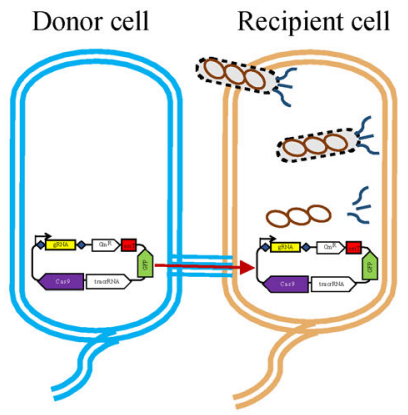

(b)

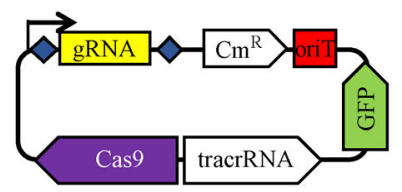

(c)
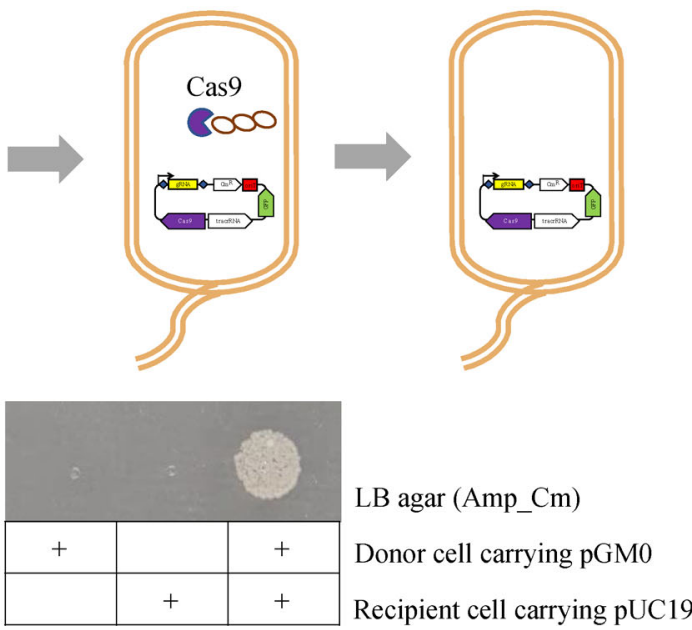

(e)

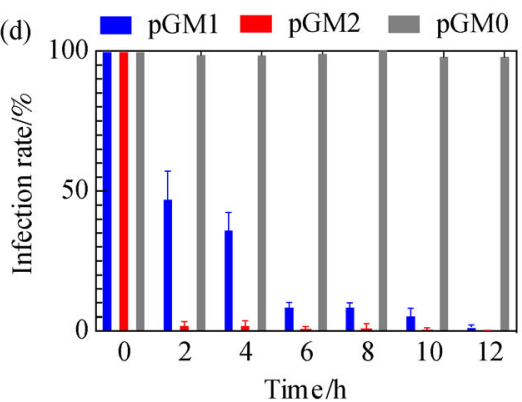

pGM1

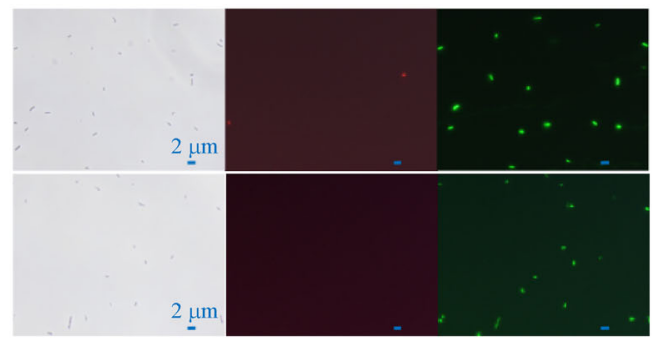

Fig. 3 MCBAS clears phage DNA in E. coli. (a) A schematic representation of MCBAS clearing MKR phage DNA in E. coli. (b) The MCBAS containing oriT for conjugative transfer. (c) The conjugation assay between donor cells (JM109) and recipient cells (XL10-Blue). JM109 with pGM0 (Cm), XL1-Blue with pUC19 (Amp) and mixture of the two strains were dropped to the LB agar plate with Cm and Amp antibiotics. (d) The MKR phage infection rate of the E. coli XL1-Blue strain infected with the MKR phage over time after transformation with pGM1 and pGM2 (Error bars represent SDs from three independent experiments). (e) Fluorescence images of the E. coli XL1-Blue strain carrying pGM1 and pGM2 for phage DNA clearance at $12 \mathrm{~h}$ in the bright field (left), red channel (middle) and green channel (right).

than pGM1. In addition, fluorescence imaging of $E$. coli carrying pGM1 and pGM2 was performed at $12 \mathrm{~h}$ to assess the states of MKR phage DNA replication and MCBAS. As shown in Fig. S5 (cf. ESM), both RFP and GFP were observed in E. coli carrying pGM1, while only GFP was observed in $E$. coli carrying pGM2, indicating pGM2 has higher prevention effect than pGM1. The results were consistent with the above phage infection rate assay.

To test the preventive effect of MCBAS with higher MKR phage concentrations, more MKR phages were used to infect $E$. coli carrying pGM1 and pGM2. As shown in Fig. 4(c), when the MOI was 1:100, the MKR infection rates of $E$. coli carrying pGM1 increased to $4 \%$ and $96.6 \%$ at 6 and $12 \mathrm{~h}$, respectively, while no significant infection was observed in E. coli carrying pGM2. As shown in Fig. 4(d), when the MOI was 1:20, the infection rates of E. coli carrying pGM1 increased to $1.75 \%$ and $100 \%$ at 4 and $12 \mathrm{~h}$, respectively. Notably, the infection rates of E. coli carrying pGM2 started to increase to $3.8 \%$ and $3.7 \%$ at 6 and $8 \mathrm{~h}$, respectively, followed by decreases to
$0.6 \%$ and $0.1 \%$ at 10 and $12 \mathrm{~h}$, respectively. When the MOI reached $1: 10$, the infection rates of $E$. coli carrying pGM2 were $3.1 \%, 12.1 \%, 10.4 \%, 1.2 \%$ and $0.1 \%$ at $4,6,8,10$ and $12 \mathrm{~h}$, respectively (Fig. 4(e)). These results indicated that a high concentration of MKR phages can accelerate the infection speed. We speculated that when the number of MKR phages in the system exceeds the threshold that pGM2 can manage, the infection rates of $E$. coli carrying pGM2 increased at the middle stage (4, 6 and $8 \mathrm{~h}$ ). However, the continuous degradation of pGM2 eventually decreased the infection rates.

Theoretically, the MCBAS targeted the MKR phage genome and generated double-strand breaks (DSBs), which caused degradation of MKR genomic DNA. To analyze why some phages can escape degradation by MCBAS, we sequenced ten MKR genomes in colonies of E. coli carrying pGM1 or pGM2 (Fig. 5). As shown in Fig. 5(a), three types of point mutations were observed in the MKR genome of $E$. coli carrying pGM1, including a $C$ to $\mathrm{G}$ mutation in the protospacer region of gRNA1 in seven 
(a)

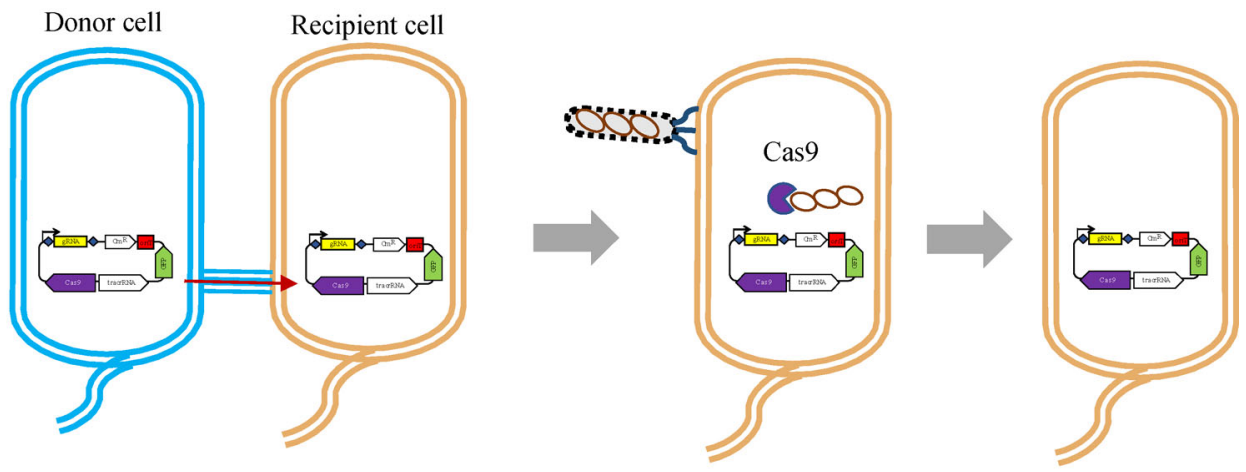

(b)

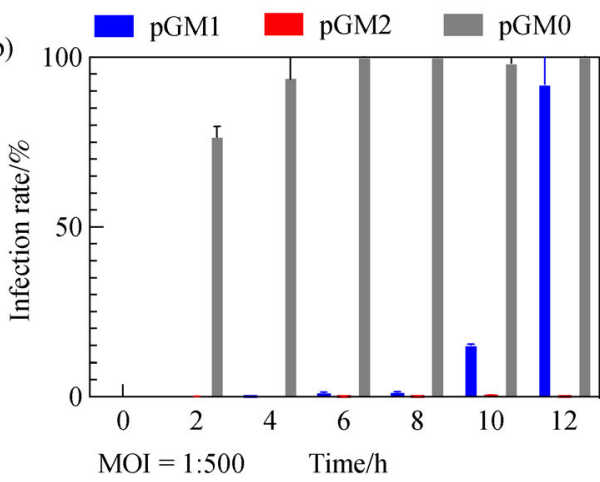

(d)

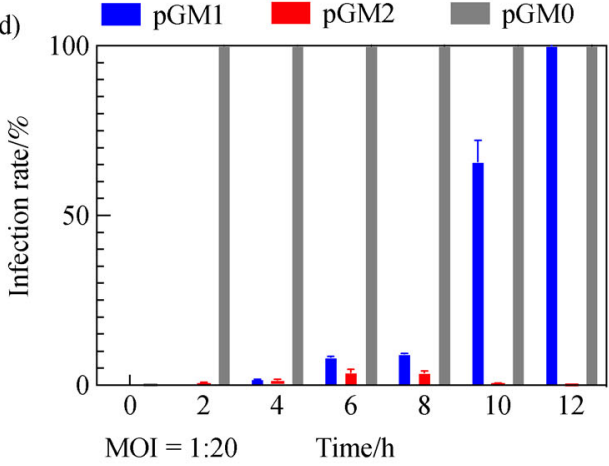

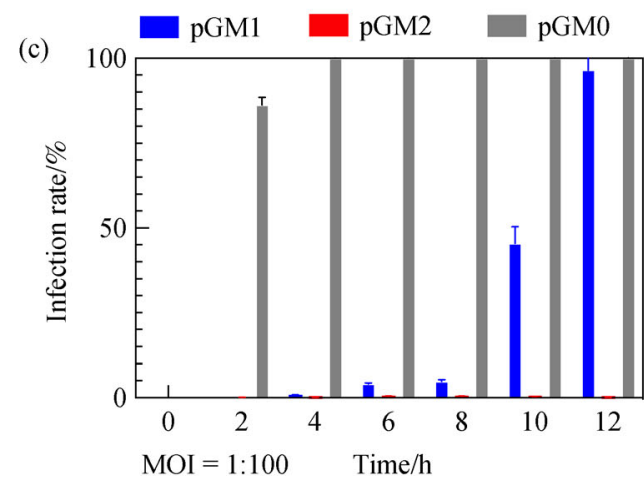

(e)

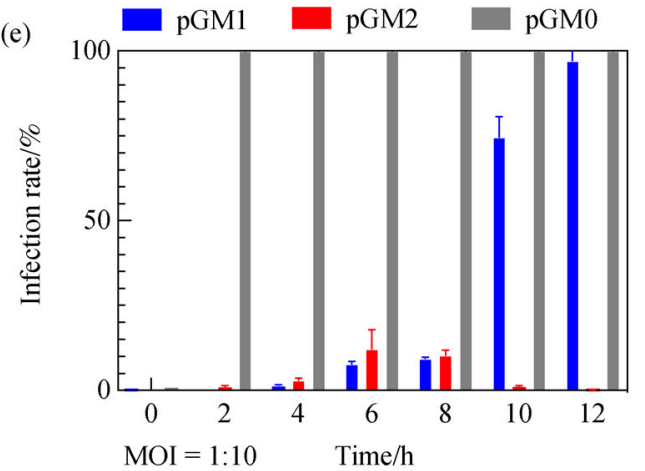

Fig. 4 MCBAS prevents phage infection of E. coli. (a) Schematic representation of MCBAS preventing MKR phage infection. The MKR phage infection rates of the $E$. coli XL1-Blue strain carrying pGM1 and pGM2 over time are shown in (b), (c), (d) and (e) when the MOI was adjusted to 1:500, 1:100, 1:20 and 1:10, respectively (Error bars represent SDs from three independent experiments).

MKR genomes and a $\mathrm{G}$ to $\mathrm{A}$ mutation in the PAM region of gRNA1 in three MKR genomes. As shown in Fig. 5(b), only one deletion mutation was observed in one MKR genome of E. coli carrying pGM2, which caused the loss of M13 replication origins.

There are two possible explanations for the escaped mutations. One is that the point mutations were spontaneously generated during phage DNA replication and then selected by CRISPR-targeting [25], the other one is that the generation of DSB in the MKR phage genome were potentially repaired through the alternative end-joining mechanism in E. coli [26]. Point mutations in PAM or protospacer regions might be generated in the process and thus cause loss of the defense function of pGM1. Compared to pGM1, pGM2 targeted two sites at the same time and thus generated two DSBs on the MKR phage genome simultaneously, which made it difficult to repair both the DSBs. Moreover, mismatch repair of the two DSBs could delete the phage replication origins, which causes loss of the replication function of the mutated MKR phage. Therefore, the MCBAS with multiple targets had a better preventive effect than that with a single target. It has been proved that the different gRNA targets potentially have different efficacy of generating DSBs. Thus, it is reasonable to improve the prevention ability of MCBAS by selecting high efficacy gRNA targets and increasing the numbers of gRNA targets.

E. coli is one of the most important cell chassis for microbial cell factories, but infection by bacteriophages in the environment may have a huge impact on its application 
(a)

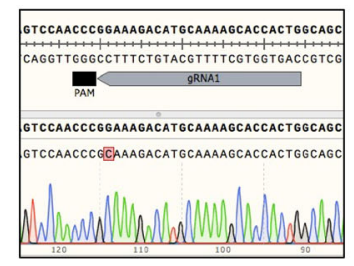

$7 / 10$ Space mutation

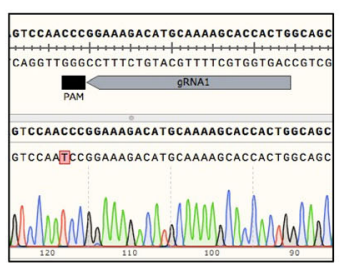

2/10 PAM mutation

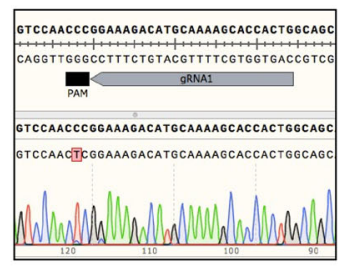

1/10 PAM mutation

(b)
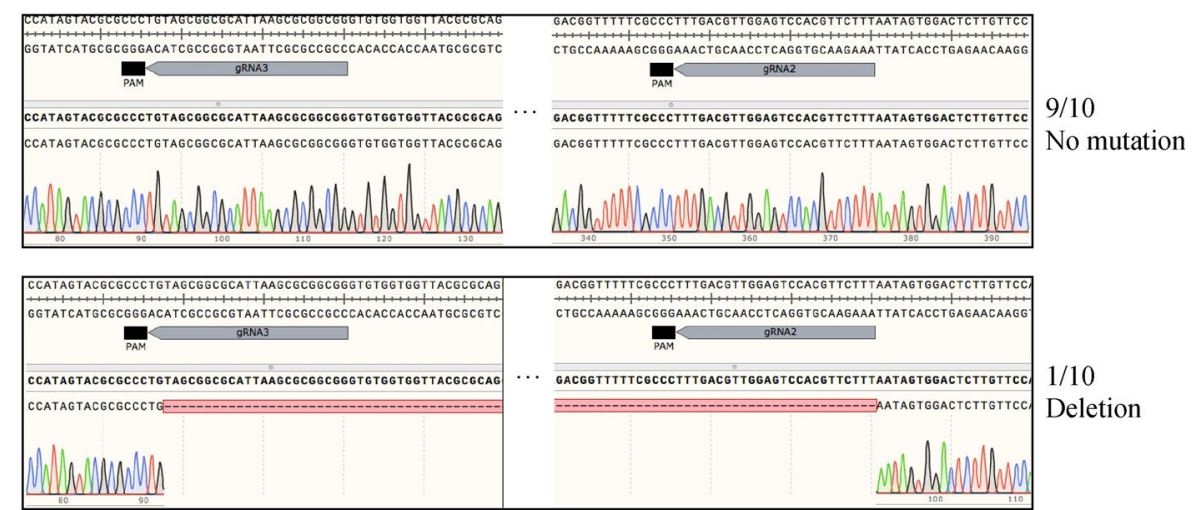

Fig. 5 Sequence verification of escaped phages. (a) Alignment of the escape mutant sequences of 10 different infected E. coli pGM1 strains; (b) alignment of the escape mutant sequences of 10 different infected E. coli pGM2 strains.

in industrial production. This study offers certain advantages. First, lytic phages, such as T4, T5 and T7, can infect most $E$. coli strains used in the lab, which increases the risk of laboratory contamination. However, the MKR phage was generated from the M13 filamentous phage, which can only infect $E$. coli without lysis of cells. Therefore, MKR phages can serve as a safe model for research on anti-phage methods. Second, we show that both pGM1 and pGM2 can effectively clear the MKR phage genome in infected E. coli. Compared with pGM1, pGM2 has a faster and better effect. This method could potentially be used to rescue industrial strains contaminated by filamentous phages or lysogenic phages through deletion of important genomic regions. Third, we show that pGM2 can achieve effective prevention with high phage yield. This method could potentially be used to study infection prevention for different kinds of phages, including filamentous phages and lytic phages. This effect is consistent with results showing protection of $E$. coli BL21 cells against T7 phage infection [27]. Fourth, traditional transformation methods, such as electroporation, limit the application of the CRISPR-Cas9 system [28]. The combination of conjugation transfer extends the industry host range with phage defense needs. Moreover, recent DNA synthesis technology makes important progress and thus promotes the development of synthetic genome [29-32], including synthetic phage genome and virus genome [33-36]. Thus it is urgently needed to develop corresponding phage defense strategy or biocontainment strategy. Our method will provide a powerful tool to defense those natural or synthesized phages. Additionally, the PAM recognition scope of the CRISPR-Cas9 system potentially limits its applications, a variety of engineered CRISPR-FnCas $12 \mathrm{a}$ proteins are generated with expanded PAM requirements [37-39]. Therefore, we anticipated combining the engineered CRISPR-FnCas12a will improve the PAM recognition scope of the MCBAS.

\section{Conclusions}

In this study, we developed MCBAS for bacteriophages defense in $E$. coli. The MCBAS can efficiently rescue $E$. coli by degrading phage DNA in vivo. The preventive effect of the MCBAS can be further improved by incorporating multiple gRNAs of different targets in the phage genome. This work establishes a synthetic biological platform for phage defense ability improvement when building microbial cell factories.

Acknowledgements The authors are grateful for the financial support from the National Key Research and Development Program of China (Grant No. 2019YFA0903800), the National Natural Science Foundation of China (Grant Nos. 31800719, 21621004), and the International (regional) cooperation and exchange projects (Grant No. 31861143017).

Electronic Supplementary Material Supplementary material is available in the online version of this article at https://dx.doi.org/10.1007/s11705-0222141-7 and is accessible for authorized users. 


\section{References}

1. Li J, Neubauer P. Escherichia coli as a cell factory for heterologous production of nonribosomal peptides and polyketides. New Biotechnology, 2014, 31(6): 579-585

2. Baeshen N A, Baeshen M N, Sheikh A, Bora R S, Ahmed M M M, Ramadan H A I, Saini K S, Redwan E M. Cell factories for insulin production. Microbial Cell Factories, 2014, 13(1): 141

3. Wang Z, Sun J, Yang Q, Yang J. Metabolic engineering Escherichia coli for the production of lycopene. Molecules (Basel, Switzerland), 2020, 25(14): 3136

4. Lemuth K, Steuer K, Albermann C. Engineering of a plasmid-free Escherichia coli strain for improved in vivo biosynthesis of astaxanthin. Microbial Cell Factories, 2011, 10(1): 29

5. Li M, Nian R, Xian M, Zhang H. Metabolic engineering for the production of isoprene and isopentenol by Escherichia coli. Applied Microbiology and Biotechnology, 2018, 102(18): 7725-7738

6. Zhao C, Zhang Y, Li Y. Production of fuels and chemicals from renewable resources using engineered Escherichia coli. Biotechnology Advances, 2019, 37(7): 107402

7. Wu H, Fan Z, Jiang X, Chen J, Chen G. Enhanced production of polyhydroxybutyrate by multiple dividing E. coli. Microbial Cell Factories, 2016, 15(1): 128

8. Chen G, Jiang X. Engineering bacteria for enhanced polyhydroxyalkanoates (PHA) biosynthesis. Synthetic and Systems Biotechnology, 2017, 2(3): 192-197

9. Liu X, Hua K, Liu D, Wu Z, Wang Y, Zhang H, Deng Z, Pfeifer B A, Jiang M. Heterologous biosynthesis of type II polyketide products using E. coli. ACS Chemical Biology, 2020, 15(5): 11771183

10. Tang Y, Wang M, Qin H, An X, Guo Z, Zhu G, Zhang L, Chen Y. Deciphering the biosynthesis of TDP- $\beta$-L-oleandrose in avermectin. Journal of Natural Products, 2020, 83(10): 3199-3206

11. Jones D T, Shirley M, Wu X Y, Keis S. Bacteriophage infections in the industrial acetone butanol (AB) fermentation process. Journal of Molecular Microbiology and Biotechnology, 2000, 2(1): 21-26

12. Chaturongakul S, Ounjai P. Phage-host interplay: examples from tailed phages and Gram-negative bacterial pathogens. Frontiers in Microbiology, 2014, 5: 442

13. Kronheim S, Daniel I M, Duan Z, Hwang S, Wong A I, Mantel I, Nodwell J R, Maxwell K L. A chemical defence against phage infection. Nature, 2018, 564(7735): 283-286

14. Abedon S T. Bacteriophage secondary infection. Virologica Sinica, 2015, 30(1): 3-10

15. Hampton H G, Watson B N J, Fineran P C. The arms race between bacteria and their phage foes. Nature, 2020, 577(7790): 327-336

16. Scholl D, Adhya S, Merril C. Escherichia coli K1's capsule is a barrier to bacteriophage T7. Applied and Environmental Microbiology, 2005, 71(8): 4872-4874

17. Vasu K, Nagaraja V. Diverse functions of restriction-modification systems in addition to cellular defense. Microbiology and Molecular Biology Reviews, 2013, 77(1): 53-72

18. Pleska M, Guet C C. Effects of mutations in phage restriction sites during escape from restriction-modification. Biology Letters, 2017,
13(12): 20170646

19. Zhou Y, Xu X, Wei Y, Cheng Y, Guo Y, Khudyakov I, Liu F, He P, Song Z, Li Z, et al. A widespread pathway for substitution of adenine by diaminopurine in phage genomes. Science, 2021, 372 (6541): 512-516

20. Barrangou R, Fremaux C, Deveau H, Richards M, Boyaval P, Moineau S, Romero D A, Horvath P. CRISPR provides acquired resistance against viruses in prokaryotes. Science, 2007, 315(5819): 1709-1712

21. Smith G P. Filamentous fusion phage: novel expression vectors that display cloned antigens on the virion surface. Science, 1985, 228 (4705): 1315-1317

22. Esvelt K M, Carlson J C, Liu D R. A system for the continuous directed evolution of biomolecules. Nature, 2011, 472(7344): 499550

23. Carlson J C, Badran A H, Guggiana N D A, Liu D R. Negative selection and stringency modulation in phage-assisted continuous evolution. Nature Chemical Biology, 2014, 10(3): 216-222

24. Bryson D I, Fan C, Guo L, Miller C, Soll D, Liu D R. Continuous directed evolution of aminoacyl-tRNA synthetases. Nature Chemical Biology, 2018, 14(2): 186

25. de Leeuw M, Baron M, David O B, Kushmaro A. Molecular insights into bacteriophage evolution toward its host. Viruses, 2020, 12(10): 1132

26. Chayot R, Montagne B, Mazel D, Ricchetti M. An end-joining repair mechanism in Escherichia coli. Proceedings of the National Academy of Sciences of the United States of America, 2010, 107(5): 2141-2146

27. Liu L, Zhao D, Ye L, Zhan T, Xiong B, Hu M, Bi C, Zhang X. A programmable CRISPR/Cas9-based phage defense system for Escherichia coli BL21(DE3). Microbial Cell Factories, 2020, 19 (1): 136

28. Dong $\mathrm{H}$, Xiang $\mathrm{H}, \mathrm{Mu} \mathrm{D}$, Wang $\mathrm{D}$, Wang $\mathrm{T}$. Exploiting a conjugative CRISPR/Cas9 system to eliminate plasmid harbouring the $\mathrm{mcr}-1$ gene from Escherichia coli. International Journal of Antimicrobial Agents, 2019, 53(1): 1-8

29. Xie Z X, Li B Z, Mitchell L A, Wu Y, Qi X, Jin Z, Jia B, Wang X, Zeng B X, Liu H M, et al. "Perfect" designer chromosome V and behavior of a ring derivative. Science, 2017, 355(6329): 1046

30. Wu Y, Li B Z, Zhao M, Mitchell L A, Xie Z X, Lin Q H, Wang X, Xiao W H, Wang Y, Zhou X, et al. Bug mapping and fitness testing of chemically synthesized chromosome X. Science, 2017, 355 (6329): 1048

31. Chen W G, Han M Z, Zhou J T, Ge Q, Wang P P, Zhang X C, Zhu S Y, Song L F, Yuan Y J. An artificial chromosome for data storage. National Science Review, 2021, 8(5): 62-70

32. Wang L, Jiang S, Chao C, He W, Wu X, Wang F, Tong T, Zou X, Li Z, Luo J, et al. Synthetic genomics: from DNA synthesis to genome design. Angewandte Chemie International Edition, 2018, 57(7): 1748-1756

33. Cello J, Paul A V, Wimmer E. Chemical synthesis of poliovirus cDNA: generation of infectious virus in the absence of natural template. Science, 2002, 297(5583): 1016-1018

34. Smith H O, Iii C A H, Pfannkoch C, Venter J C. Generating a synthetic genome by whole genome assembly: X174 bacteriophage from synthetic oligonucleotides. Proceedings of the National 
Academy of Sciences of the United States of America, 2003, 100 (26): 15440-15445

35. Chan L Y, Kosuri S, Endy D. Refactoring bacteriophage T7. Molecular Systems Biology, 2005, 1: 2005.0018

36. Thao T, Labroussaa F, Ebert N, Kovski P V, Thiel V. Rapid reconstruction of SARS-CoV-2 using a synthetic genomics platform. Nature, 2020, 582(7813): 561-565

37. Zhang J, Zhang D, Zhu J, Liu H, Liang S, Luo Y. Efficient multiplex genome editing in Streptomyces via engineered CRISPR-Cas12a systems. Frontiers in Bioengineering and Biotechnology, 2020, 8: 726

38. Wang L, Wang H, Liu H, Zhao Q, Liu B, Wang L, Zhang J, Zhu J, Bao R, Luo Y. Improved CRISPR-Cas12a-assisted one-pot DNA editing method enables seamless DNA editing. Biotechnology and Bioengineering, 2019, 116(6): 1463-1474

39. Liu H, Wang L, Luo Y. Blossom of CRISPR technologies and applications in disease treatment. Synthetic and Systems Biotechnology, 2018, 3(4): 217-228 